\title{
Cerebral Ischemia is Associated With Corneal Nerve Loss and Brain Atrophy in Mild Cognitive Impairment and Dementia
}

\section{Georgios Ponirakis}

Weill Cornell Medicine - Qatar

Ahmed Elsotouhy

Hamad Medical Corporation

Hanadi Al Hamad

Hamad Medical Corporation

\section{Surjith Vattoth}

University of Arkansas for Medical Sciences

loannis N. Petropoulos

Weill Cornell Medicine - Qatar

Adnan Khan

Weill Cornell Medicine - Qatar

Hoda Gad

Weill Cornell Medicine - Qatar

Fatima Al-Khayat

Weill Cornell Medicine - Qatar

Mani Chandran

Hamad Medical Corporation

Marwan Ramadan

Hamad Medical Corporation

Marwa Elorrabi

Hamad Medical Corporation

Masharig Gadelseed

Hamad Medical Corporation

Rhia Tosino

Hamad Medical Corporation

Priya V. Gawhale

Hamad Medical Corporation

Maryam Alobaidi

Hamad Medical Corporation

Shafi Khan 
Hamad Medical Corporation

Pravija Manikoth

Hamad Medical Corporation

Yasmin Hamdi

Hamad Medical Corporation

Noushad Thodi

Hamad Medical Corporation

Hamad Almuhannadi

Weill Cornell Medicine - Qatar

Salma Al-Mohannadi

Weill Cornell Medicine - Qatar

Fatema AIMarri

Weill Cornell Medicine - Qatar

Murtaza Qazi

Weill Cornell Medicine - Qatar

Ahmed Own

Hamad Medical Corporation

Ziyad R. Mahfoud

Weill Cornell Medicine - Qatar

Ashfaq Shuaib

University of Alberta

Rayaz A Malik ( $\sim$ ram2045@qatar-med.cornell.edu )

University of Manchester https://orcid.org/0000-0002-7188-8903

\section{Research}

Keywords: Ischemic lesions, surrogate marker, corneal confocal microscopy, corneal nerve fibers, brain atrophy, dementia, mild cognitive impairment

Posted Date: November 5th, 2020

DOI: https://doi.org/10.21203/rs.3.rs-101841/v1

License: (9) This work is licensed under a Creative Commons Attribution 4.0 International License. Read Full License 


\section{Abstract}

\section{Background}

The prevalence of cerebral ischemia increases with age and is a risk factor for cognitive impairment and dementia. This study assessed the association of brain ischemic lesions with the severity of neurodegeneration utilizing brain volumetric MRI and corneal confocal microscopy (CCM) in patients with mild cognitive impairment $(\mathrm{MCl})$ and dementia.

\section{Methods}

Subjects with $\mathrm{MCl}$ and dementia without diabetes underwent cognitive screening, CCM, assessment of ischemic lesions and quantitative brain MRI.

Results

Of 63 subjects with $\mathrm{MCl}(n=44)$ and dementia $(n=19), 11$ had no ischemia, 32 had subcortical ischemia and 20 had both cortical and subcortical ischemia. Subjects with $\mathrm{MCl}$ and dementia had comparable percentage of cerebral ischemia $(P=0.25)$. Global cognitive function was significantly impaired in subjects with both cortical and subcortical ischemia $(P<0.05)$ but not in those with subcortical ischemia $(P=0.10)$ compared to those without ischemia. Corneal nerve fiber density (CNFD) $(P<0.01)$, branch density $(C N B D)(P<0.05)$ and fiber length $(C N F L)(P<0.01)$ were significantly lower in subjects with both cortical and subcortical ischemia compared to those without ischemia and CNFD $(P<0.05)$, CNBD $(P<0.05)$ and $C N F L(P<0.05)$ were significantly lower in subjects with both cortical and subcortical ischemia compared to those with subcortical ischemia. In subjects with both cortical and subcortical ischemia whole brain $(P<0.01)$ and hippocampal volume $(P<0.001)$ were significantly lower and ventricle volume was higher $(P<0.05)$ compared to those without ischemia and hippocampal volume $(P<0.01)$ was lower and ventricle volume was higher $(P<0.01)$ in subjects with both cortical and subcortical ischemia compared to those with subcortical ischemia.

\section{Conclusions}

The presence of cortical and subcortical ischemia is associated with cognitive impairment, corneal nerve loss and brain atrophy in patients with $\mathrm{MCl}$ and dementia.

\section{Background}

Cerebrovascular ischemic lesions in the brain are present in $11-94 \%$ of people aged $\geq 60$ years [1] and increase the risk of cognitive impairment [2] and dementia [3, 4], depending on the person's age, education and lifestyle, as well as the location and size of ischemic load [5]. Ischemic lesions are incomplete infarctions due to reduced blood flow caused by critical stenosis in small vessels in the white matter of the brain [5] and can increase and decrease in size [6]. Management of hypertension [7, 8] and diabetes $[9,10]$, antiplatelet therapy [11] or use of calcium-channel blockers [12] may prevent or reduce ischemic 
lesions. There is limited evidence on the relationship between the presence and severity of ischemic lesions and neurodegeneration $[13,14]$.

Patients with ischemic lesions and lacunar stroke have impaired executive function and reduced brain volume [13]. Corneal confocal microscopy (CCM) has shown significant corneal nerve loss in patients with TIA [15] and acute ischemic stroke [16]. Furthermore, corneal nerve loss has been associated with the presence of ischemic lesions in patients with acute ischemic stroke after adjusting for age, diabetes, gender, dyslipidemia and smoking [14]. We have also previously reported that patients with mild cognitive impairment $(\mathrm{MCl})$ and dementia have significant corneal nerve loss which is associated with the severity of cognitive impairment and disability $[17,18]$.

The aim of this study was to assess the association between the presence and severity of cerebral ischemic lesions with neurodegeneration quantified by brain volumetric MRI and CCM in patients with $\mathrm{MCl}$ and dementia, including Alzheimer's disease (AD), vascular dementia (VaD) and mixed dementia. The severity of neurodegeneration was compared between patients without and with ischemic lesions in the subcortex and both subcortex and cortex. This study excluded those with diabetes as this is a confounding factor for corneal nerve loss [19].

\section{Methods}

Patients with $\mathrm{MCl}$, dementia, including $A D, V a D$ and mixed $A D$ aged 60-85 years old were recruited from the Geriatric and Memory clinic in Rumailah Hospital, Doha, Qatar between 18/09/16 and 31/07/19. Patients with severe anxiety, severe depression, Parkinson's disease, frontotemporal dementia and Lewy body dementia, hypomania, and severe dementia who were unable to cooperate were excluded. Additionally, patients with peripheral neuropathy including diabetes, vitamin $\mathrm{B}_{12}$ deficiency, hypothyroidism, HIV infection and hepatitis $\mathrm{C}$ were excluded. Patients with dry eyes, corneal dystrophies, ocular trauma or surgery in the preceding 12 months were excluded. This study was approved by the Institutional Review Board of Weill Cornell Medicine in Qatar and Hamad Medical Corporation and all participants gave informed consent to take part in the study. The research adhered to the tenets of the declaration of Helsinki.

\section{Demographic and metabolic measures}

Age, gender, ethnicity, blood pressure, weight, body mass index (BMI), HbA1c, cholesterol, triglycerides, thyroid stimulating hormone (TSH), free thyroxine (FT4) and vitamin $\mathrm{B}_{12}$ were recorded.

\section{Cognitive screening}

Cognitive screening was performed using the Montreal Cognitive Assessment (MoCA) test. The MoCA assesses seven cognitive domains including visuospatial/executive, naming, memory, attention, language, abstraction and delayed recall giving a total score of 30 . A score of $\otimes 26$ indicates cognitive 
impairment. A point was added for individuals who had formal education $\leq 6$ th grade. Cognitive symptom duration was estimated from the clinical history obtained from relatives and participants.

\section{Diagnosis}

The diagnosis of $\mathrm{MCl}$ and dementia, including $A D, V a D$ and mixed $A D$ were based on the ICD-10 criteria [20]. The diagnosis was made according to consensus decision by geriatricians, geriatric psychiatrists and neurologists to exclude reversible, complex and young-onset dementia. The diagnoses of $\mathrm{MCl}$ and dementia were based on a patient history and examination, which include (1) presenting complaint and history of illness; (2) comprehensive history of each of the cognitive domains using MoCA; (3) psychiatric history for ruling out depression, mood disorders, and psychosis; (4) medical history including episodes of delirium and other medical comorbidities; (5) medication history; (6) functional history of basic daily living activities. A comprehensive organic work-up including blood tests and brain imaging was undertaken to exclude other potentially reversible causes of cognitive decline such as tumors, subdural hematoma or normal pressure hydrocephalus. The diagnosis of $A D$ was based on typical features of $A D$ on MRI, including volume loss of hippocampi, entorhinal cortex, and amygdala. The diagnosis of mixed $A D$ was based on the presence of $A D$ and significant vascular changes. The diagnosis of probable or possible $\mathrm{VaD}$ was based on multiple large vessel infarcts or a single strategically placed infarct in the angular gyrus, thalamus, basal forebrain, or posterior (PCA) or anterior cerebral artery (ACA) territories, and multiple lacunes in basal ganglia and white matter, extensive periventricular white matter lesions or combinations thereof.

\section{Brain MRI acquisition}

MRI was performed on a superconductive magnet operated at 3T (Skyra, Siemens) at the MRI unit in Rumailah Hospital. The subject's head was immobilized with a head holder to minimize motion artifacts. A T1-weighted 3D magnetisation prepared rapid acquisition gradient echo sequence (MPRAGE) was obtained in the sagittal plane with a $1 \mathrm{~mm}$ slice thickness, repetition time of $1900 \mathrm{~ms}$, echo time of 2.67 $\mathrm{ms}$ and $2.46 \mathrm{~ms}$, inversion time of $1100 \mathrm{~ms}$ and $900 \mathrm{~ms}$, flip angle of 9 degree and 15 degree, and FOV= $240 \times 100$. Coronal and axial reformatted MPRAGE images are reconstructed from the sagittal 3D sequence.

\section{Ischemic lesion assessment}

The presence of ischemic lesions was defined as hyperintense foci on T2 and FLAIR. Small vessel disease (SVD) was assessed by the presence of white matter hyperintensities (WMH) in cortical, subcortical or both regions. Infarcts including lacunes, large infarcts and hemorrhage were not included in the analysis. Foci that were hyperintense on T2 and showed central low signal with a peripheral rim of hyperintensity on FLAIR were defined as lacunes. Larger areas of gliosis/encephalomalacia following a vascular pattern or diffusion restricting acute ischemic lesions were defined as infarcts. Subcortical ischemia was based on the presence of ischemic lesions located in the subcortical white matter, deep 
grey nuclei including basal ganglia, thalami, and mesial temporal lobe. Cortical ischemia was based on the presence of ischemic lesions located in the cerebral convexity cortex.

\section{Brain volume analysis}

MRI T1-weighted 3D MPRAGE sequences were processed using NeuroQuant (NQ), an FDA approved fully automated software $[21,22]$ to measure brain volumes. The brain volume was adjusted for percentage of intracranial volume (ICV) which includes all segmented structures to minimize the impact of the head size as a confounding factor. The ICV percentage of the whole brain, cortical gray matter, ventricle, hippocampi, frontal, temporal and parietal lobe are included in this study.

\section{Corneal confocal microscopy}

CCM analysis was performed with the Heidelberg Retinal Tomograph III Rostock Cornea Module (Heidelberg Engineering $\mathrm{GmbH}$, Heidelberg, Germany). The cornea was locally anesthetized by instilling 1 drop of $0.4 \%$ benoxinate hydrochloride (Chauvin Pharmaceuticals, Chefaro, UK) and Viscotears gel (Carbomer 980, 0.2\%, Novartis, UK) was used as the coupling agent between the cornea and the TomoCap as well as between the TomoCap and the objective lens. Subjects were instructed to fixate on a target with the eye not being examined. Several scans of the sub-basal nerve plexus in the central cornea were captured per eye for $\sim 2 \mathrm{~min}$. The field of view of each image is $400 \mathrm{X} 400 \mu \mathrm{m}$. At a separate time, three high clarity images per eye were selected by one researcher blind to the patient diagnosis using established criteria based on depth, focus position and contrast [23]. Corneal nerve fiber density (CNFD) (fibers $/ \mathrm{mm}^{2}$ ), branch density (CNBD) (branches $/ \mathrm{mm}^{2}$ ) and fiber length (CNFL) (total fiber length $\mathrm{mm} / \mathrm{mm}^{2}$ ) were quantified manually using CCMetrics, a validated image analysis software [24].

\section{Statistical analysis}

Given that the difference in neuronal injury measured by brain volumetric MRI and CCM between subjects without and with ischemic lesions in the subcortex and both subcortex and cortex have not been studied before, the results were analysed as an exploratory study and not adjusted for multiple testing or multiple comparisons [25].

Variables were summarized using means and standard deviations for numeric variables and frequency distribution for categorical variables. Variables were compared between subjects without ischemic lesions, with subcortical ischemia and both subcortical and cortical ischemia using one-way analysis of variance (ANOVA) with least significant difference (LSD) post hoc test for pairwise comparisons and categorical outcomes were compared using Chi-square test. The age-adjusted mean difference in the volume of different brain structures and corneal nerve measures between the three groups were estimated using covariance (ANCOVA) with LSD test for post hoc comparisons.

All analyses were performed using IBM-SPSS (version 26; SPSS Inc, Armonk NY). A two-tailed P value of $\leq 0.05$ was considered significant. 


\section{Results}

\section{Demographic and clinical characteristics (Table 1)}

63 subjects with $\mathrm{MCl}(n=44)$ and dementia $(n=19)$ were studied. The clinical characteristics of those without ischemia $(n=11)$, subcortical ischemia $(n=32)$ and both cortical and subcortical ischemia $(n=20)$ are summarized in Table 1. Gender $(P=0.17)$, and the percentage of subjects with hypertension $(P=0.51)$, $\mathrm{MCl}$ and dementia $(P=0.25)$ were comparable between the three groups. Subjects without ischemia were significantly younger compared to those with subcortical ischemia $(P<0.05)$ and both cortical and subcortical ischemia $(P<0.01)$, but age was comparable between the latter two groups $(P=0.30)$. The duration of cognitive impairment, systolic (SBP) and diastolic blood pressure (DBP), HbA1c, total cholesterol, and triglycerides were comparable between the three groups. Body weight and BMI were lower in subjects with both cortical and subcortical ischemia compared to subjects with subcortical ischemia $(P=0.05)$ and without ischemia $(P<0.05)$, respectively.

\section{Cognitive function (Table 2)}

Based on the MoCA, global cognitive function was significantly lower in subjects with both cortical and subcortical ischemia $(P<0.05)$, but not those with subcortical ischemia when compared to subjects without ischemia $(P=0.28)$. A lesser percentage of subjects with ischemia completed successfully the tests for visuospatial executive function $(22.2 \%$ and $11.8 \%$ vs $63.6 \%, \mathrm{P}<0.05)$ and orientation $(44.4 \%$ and $29.4 \%$ vs $81.8 \%, P=0.01$ ) were lower in subjects with ischemia compared to those without ischemia. The percentage of subjects with ischemia who successfully completed the attention test was nonsignificantly lower compared to those without ischemia ( $37.0 \%$ and $35.3 \%$ vs $72.7 \%, P=0.63$ ). Performance in the other domains, including naming, sentence repetition and letter fluency, abstraction to connect related concepts and delayed recall memory test were comparable between all three groups.

\section{Corneal nerve fiber measures (Table 2 \& Figure 1)}

There was no difference in corneal nerve measures between subjects with subcortical ischemia compared to those without ischemia. Corneal nerve fiber density (CNFD) $(P<0.01)$, branch density (CNBD) $(P<0.05)$ and fiber length $(C N F L)(P<0.01)$ were significantly lower in subjects with both cortical and subcortical ischemia compared to those without ischemia and CNFD $(P<0.05), C N B D(P<0.05)$ and CNFL $(P<0.05)$ were significantly lower in subjects with both cortical and subcortical ischemia compared to those with subcortical ischemia. After adjusting for age, subjects with both cortical and subcortical ischemia had a significantly lower CNFD $\left(-7.2\right.$ fibers $\left./ \mathrm{mm}^{2}, 95 \% \mathrm{Cl}-14.1--0.4, \mathrm{P}<0.05\right)$ compared to those without ischemia and a significantly lower CNFL $\left(-4.0 \mathrm{~mm} / \mathrm{mm}^{2}, 95 \% \mathrm{Cl}-7.5--0.5, \mathrm{P}<0.05\right)$ compared to those with subcortical ischemia. There was no significant difference in the CNBD:CNFD ratio between the three groups.

\section{Volumetric brain MRI (Table 2 \& Figure 2)}


Whole brain volume was significantly lower in subjects with subcortical ischemia compared to those without ischemia $(P<0.05)$. Whole brain $(P<0.01)$ and hippocampal volume $(P<0.001)$ were significantly lower and ventricular volume was higher $(P<0.05)$ in subjects with both cortical and subcortical ischemia compared to those without ischemia and hippocampal volume $(P<0.01)$ was significantly lower and ventricular volume was higher $(P<0.01)$ in subjects with both cortical and subcortical ischemia compared to those with subcortical ischemia. After adjusting for age, the significant difference in whole brain volume between those with and without ischemia was lost $(P=0.15-0.52)$, whereas subjects with cortical and subcortical ischemia had a significantly lower hippocampal volume $(-0.06 \mathrm{ICV} \%, 95 \% \mathrm{Cl}-0.10--0.02$, $\mathrm{P}<0.01)$ and larger ventricular volume $(1.27 \mathrm{ICV} \%, 95 \% \mathrm{Cl} 0.25-2.29, \mathrm{P}<0.05)$ compared to those with subcortical ischemia but not those without ischemia ( $P=0.08$ and 0.14 , respectively). There was no significant difference in cortical gray matter, frontal, temporal, and parietal lobe volumes between the three groups.

\section{Peripheral neuropathy assessments (Table 2)}

Vibration perception threshold (VPT) and sudomotor function measured by electrochemical skin conductance (ESC) on the feet, were comparable between all three groups $(P=0.10)$, even after adjusting for age $(P=0.82)$.

\section{Discussion}

In this study, cortical and subcortical ischemia was associated with neurodegeneration quantified by brain volumetric MRI and corneal confocal microscopy (CCM) in patients with $\mathrm{MCl}$ and dementia. It was also associated with reduced global cognitive function, particularly executive function and orientation.

Unlike vascular dementia $(\mathrm{VaD})$ caused by multiple or strategic infarction or hemorrhage, which develops relatively quickly in patients with stroke, mixed dementia caused by amyloid deposits and ischemic lesions develops relatively slowly $[26,27]$. Ischemic lesions arise as a consequence of chronically reduced blood flow to the white matter caused by critical stenosis of the cortical medullary branches [5] and are present in approximately $50 \%$ of patients with Alzheimer's disease (AD) [28]. Indeed, ischemic lesions can be present in $11-94 \%$ of people aged $\geq 60$ years [1] and can increase in size, shrink or in rare instances, disappear [6].

There is a need for reliable surrogate biomarkers to identify patients at a higher risk for cognitive impairment and dementia. Whilst ischemic lesions have been associated with an increase in the risk of cognitive impairment [2] and dementia [3, 4], there is scarce evidence on their association with neurodegeneration $[13,14]$. Brain volumetric MRI has been suggested as a surrogate marker of neurodegeneration [13] especially as brain atrophy is associated with impairment in executive function in patients with ischemic lesions [13]. This cross-sectional study shows that ischemic lesions are associated with impaired executive function and orientation in patients with $\mathrm{MCl}$ and dementia. Furthermore, brain volumetric MRI showed significantly reduced hippocampal volume and increased 
ventricular volume in patients with both cortical and subcortical ischemia compared to those with subcortical ischemia after adjusting for age.

This study also utilized CCM to assess if corneal nerve loss could act as a surrogate marker for the extent of cerebral ischemia. Recently, we showed that reduced corneal nerve fiber density was associated with ischemic lesions in patients with acute ischemic stroke after adjusting for age, diabetes, gender, dyslipidemia and smoking [14]. We have demonstrated significant corneal nerve loss in patients with $\mathrm{MCl}$ and dementia, which was associated with the severity of cognitive impairment and disability [17]. Moreover we have recently shown that the diagnostic accuracy of CCM was high and comparable with MRI based medial temporal lobe atrophy (MTA) rating for dementia but was superior in $\mathrm{MCl}$ [18]. This study now shows that patients with both cortical and subcortical ischemia have a significantly lower CNFD compared to those without ischemia, and significantly lower CNFL compared to those with subcortical ischemia after adjusting for. There was no significant difference in the corneal nerve measures between patients with subcortical compared to no ischemia, suggesting that more widespread ischemia is associated with corneal nerve degeneration in patients with $\mathrm{MCl}$ and dementia.

The prevalence of ischemic lesions increases with age [29], hypertension $[7,8]$ and diabetes $[9,10]$ and they may improve with antiplatelet therapy [11] and improved management of hypertension $[7,8]$ and diabetes $[9,10]$. These same risk factors have been related to corneal nerve degeneration [30] and indeed improvement in blood pressure, lipids and glycemic control is associated with corneal nerve regeneration $[31,32]$.

A significant limitation of this study is the small sample size due to the need to exclude patients with diabetes, a confounding factor for corneal nerve loss.

\section{Conclusions}

This study shows that cortical and subcortical ischemia was associated with impaired global cognitive function, corneal nerve fiber loss and brain atrophy in patients with $\mathrm{MCl}$ and dementia. CCM and brain volumetric MRI may act as non-invasive surrogate markers of neurodegeneration associated with cortical and subcortical ischemia in patients with $\mathrm{MCl}$ and dementia. Further longitudinal studies are needed to evaluate the utility of CCM as a surrogate marker of neurodegeneration and progressive cognitive dysfunction.

\section{Abbreviations}

AD: Alzheimer's disease

BMI: body mass index

CCM: corneal confocal microscopy 
CNBD: corneal nerve branch density

CNFD: corneal nerve fiber density

CNFL: corneal nerve fiber length

ESC: electrochemical skin conductance

FT4: free thyroxine

ICV: intracranial volume

MCl: mild cognitive impairment

MoCA: Montreal Cognitive Assessment

MPRAGE: magnetization prepared rapid acquisition gradient echo sequence

MTA: medial temporal lobe atrophy

TSH: thyroid stimulating hormone

VaD: vascular dementia

VPT: Vibration perception threshold

\section{Declarations}

\section{Ethics approval and consent to participate}

Informed consent was obtained for all subjects according to the Declaration of Helsinki (1991), and protocols and procedures were approved by the Institutional Review Board of Weill Cornell Medicine in Qatar and Hamad Medical Corporation.

\section{Consent for publication}

All contributing authors have given their consent for the publication of this study.

\section{Availability of data and materials}

Data are available from the authors upon reasonable request.

\section{Competing interests}


The authors declare that they have no competing interests.

\section{Funding}

This work was funded by the Qatar National Research Fund (BMRP-5726113101 \& NPRP12S-0213190080).

\section{Authors' contributions}

RAM and GP had full access to all the data in the study and take responsibility for the integrity of the data and the accuracy of the data analysis.

Study concept and design: AE, GP and RAM.

Acquisition, analysis, or interpretation of data: All authors.

Drafting of the manuscript: GP and RAM.

Critical revision of the manuscript for important intellectual content: All authors.

Statistical analysis: GP, ZM and RAM.

Obtained funding: RAM.

Administrative, technical, or material support: All authors.

All authors have read and approved the final manuscript.

\section{Acknowledgements}

We thank Dr Hanadi Al-Hamad, the Medical Director of Rumailah Hospital and Qatar Rehabilitation Institute and her staff at Rumailah Hospital for providing the facility and helping to undertake this study. We particularly thank all the participants and their relatives for their efforts, will and commitment to be involved in the study. We also thank the WCM-Q Clinical Research Core for statistical advice.

\section{References}

1. Debette S, Markus HS: The clinical importance of white matter hyperintensities on brain magnetic resonance imaging: systematic review and meta-analysis. BMJ 2010, 341:c3666.

2. Mungas D, Jagust WJ, Reed BR, Kramer JH, Weiner MW, Schuff N, Norman D, Mack WJ, Willis L, Chui HC: MRI predictors of cognition in subcortical ischemic vascular disease and Alzheimer's disease. Neurology 2001, 57(12):2229-2235. 
3. Debette S, Beiser A, DeCarli C, Au R, Himali JJ, Kelly-Hayes M, Romero JR, Kase CS, Wolf PA, Seshadri $S$ : Association of MRI markers of vascular brain injury with incident stroke, mild cognitive impairment, dementia, and mortality: the Framingham Offspring Study. Stroke 2010, 41(4):600-606.

4. Prins ND, van Dijk EJ, den Heijer T, Vermeer SE, Koudstaal PJ, Oudkerk M, Hofman A, Breteler MM: Cerebral white matter lesions and the risk of dementia. Arch Neurol 2004, 61(10):1531-1534.

5. Roh JH, Lee JH: Recent updates on subcortical ischemic vascular dementia. J Stroke 2014, 16(1):1826.

6. Wardlaw JM, Valdes Hernandez MC, Munoz-Maniega S: What are white matter hyperintensities made of? Relevance to vascular cognitive impairment. J Am Heart Assoc 2015, 4(6):001140.

7. de Leeuw FE, de Groot JC, Oudkerk M, Witteman JC, Hofman A, van Gijn J, Breteler MM: Hypertension and cerebral white matter lesions in a prospective cohort study. Brain 2002, 125(Pt 4):765-772.

8. Dufouil C, de Kersaint-Gilly A, Besancon V, Levy C, Auffray E, Brunnereau L, Alperovitch A, Tzourio C: Longitudinal study of blood pressure and white matter hyperintensities: the EVA MRI Cohort. Neurology 2001, 56(7):921-926.

9. McNay EC: The impact of recurrent hypoglycemia on cognitive function in aging. Neurobiol Aging 2005, 26 Suppl 1:76-79.

10. Geijselaers SLC, Sep SJS, Claessens D, Schram MT, van Boxtel MPJ, Henry RMA, Verhey FRJ, Kroon AA, Dagnelie PC, Schalkwijk CG et al: The Role of Hyperglycemia, Insulin Resistance, and Blood Pressure in Diabetes-Associated Differences in Cognitive Performance-The Maastricht Study. Diabetes Care 2017, 40(11):1537-1547.

11. Gorelick PB, Scuteri A, Black SE, Decarli C, Greenberg SM, ladecola C, Launer LJ, Laurent S, Lopez OL, Nyenhuis D et al: Vascular contributions to cognitive impairment and dementia: a statement for healthcare professionals from the american heart association/american stroke association. Stroke 2011, 42(9):2672-2713.

12. Pantoni L, Bianchi C, Beneke M, Inzitari D, Wallin A, Erkinjuntti T: The Scandinavian Multi-Infarct Dementia Trial: a double-blind, placebo-controlled trial on nimodipine in multi-infarct dementia. $J$ Neurol Sci 2000, 175(2):116-123.

13. Nitkunan A, Lanfranconi S, Charlton RA, Barrick TR, Markus HS: Brain atrophy and cerebral small vessel disease: a prospective follow-up study. Stroke 2011, 42(1):133-138.

14. Kamran S, Khan A, Salam A, Akhtar N, Petropoulos I, Ponirakis G, Babu B, George P, Shuaib A, Malik RA: Cornea: A Window to White Matter Changes in Stroke; Corneal Confocal Microscopy a Surrogate Marker for the Presence and Severity of White Matter Hyperintensities in Ischemic Stroke. J Stroke Cerebrovasc Dis 2020:104543.

15. Gad H, Khan A, Akhtar N, Kamran S, El-Sotouhy A, Dargham SR, Petropoulos IN, Ponirakis G, Shuaib A, Streletz LJ et al: Correction: Corneal nerve and endothelial cell damage in patients with transient ischemic attack and minor ischemic stroke. PLoS One 2019, 14(5):e0217672.

16. Khan A, Kamran S, Akhtar N, Ponirakis G, Al-Muhannadi H, Petropoulos IN, Al-Fahdawi S, Qahwaji R, Sartaj F, Babu B et al: Corneal Confocal Microscopy detects a Reduction in Corneal Endothelial Cells 
and Nerve Fibres in Patients with Acute Ischemic Stroke. Sci Rep 2018, 8(1):17333.

17. Ponirakis G, Al Hamad H, Sankaranarayanan A, Khan A, Chandran M, Ramadan M, Tosino R, Gawhale PV, Alobaidi M, AISulaiti E et al: Association of corneal nerve fiber measures with cognitive function in dementia. Ann Clin Trans/ Neurol 2019, 6(4):689-697.

18. Al-Janahi E, Ponirakis G, Al Hamad H, Vattoth S, Elsotouhy A, Petropoulos IN, Khan A, Gad H, Chandran M, Sankaranarayanan A et al: Corneal Nerve and Brain Imaging in Mild Cognitive Impairment and Dementia. J Alzheimers Dis 2020.

19. Tavakoli M, Quattrini C, Abbott C, Kallinikos P, Marshall A, Finnigan J, Morgan P, Efron N, Boulton AJ, Malik RA: Corneal confocal microscopy: a novel noninvasive test to diagnose and stratify the severity of human diabetic neuropathy. Diabetes Care 2010, 33(8):1792-1797.

20. Organization WH: The ICD-10 classification of mental and behavioural disorders : clinical descriptions and diagnostic guidelines.Geneva: World Health Organization 1992.

21. Brewer JB, Magda S, Airriess C, Smith ME: Fully-automated quantification of regional brain volumes for improved detection of focal atrophy in Alzheimer disease. AJNR Am J Neuroradio/2009, $30(3): 578-580$.

22. Stelmokas J, Yassay L, Giordani B, Dodge HH, Dinov ID, Bhaumik A, Sathian K, Hampstead BM: Translational MRI Volumetry with NeuroQuant: Effects of Version and Normative Data on Relationships with Memory Performance in Healthy Older Adults and Patients with Mild Cognitive Impairment. J Alzheimers Dis 2017, 60(4):1499-1510.

23. Kalteniece A, Ferdousi M, Adam S, Schofield J, Azmi S, Petropoulos I, Soran H, Malik RA: Corneal confocal microscopy is a rapid reproducible ophthalmic technique for quantifying corneal nerve abnormalities. PLoS One 2017, 12(8):e0183040.

24. Dabbah MA, Graham J, Petropoulos IN, Tavakoli M, Malik RA: Automatic analysis of diabetic peripheral neuropathy using multi-scale quantitative morphology of nerve fibres in corneal confocal microscopy imaging. Medical image analysis 2011, 15(5):738-747.

25. Rothman KJ: No adjustments are needed for multiple comparisons. Epidemiology 1990, 1(1):43-46.

26. Pendlebury ST, Rothwell PM: Prevalence, incidence, and factors associated with pre-stroke and poststroke dementia: a systematic review and meta-analysis. Lancet Neuro/ 2009, 8(11):1006-1018.

27. Pendlebury ST: Stroke-related dementia: rates, risk factors and implications for future research. Maturitas 2009, 64(3):165-171.

28. Wang BW, Lu E, Mackenzie IR, Assaly M, Jacova C, Lee PE, Beattie BL, Hsiung GY: Multiple pathologies are common in Alzheimer patients in clinical trials. Can J Neurol Sci 2012, 39(5):592599.

29. Smith EE, Saposnik G, Biessels GJ, Doubal FN, Fornage M, Gorelick PB, Greenberg SM, Higashida RT, Kasner SE, Seshadri S et al: Prevention of Stroke in Patients With Silent Cerebrovascular Disease: A Scientific Statement for Healthcare Professionals From the American Heart Association/American Stroke Association. Stroke 2017, 48(2):e44-e71. 
30. Ponirakis G, Petropoulos IN, Alam U, Ferdousi M, Asghar O, Marshall A, Azmi S, Jeziorska M, Mahfoud ZR, Boulton AJM et al: Hypertension Contributes to Neuropathy in Patients With Type 1 Diabetes. Am J Hypertens 2019, 32(8):796-803.

31. Tavakoli M, Kallinikos P, Iqbal A, Herbert A, Fadavi H, Efron N, Boulton AJ, R AM: Corneal confocal microscopy detects improvement in corneal nerve morphology with an improvement in risk factors for diabetic neuropathy. Diabet Med 2011, 28(10):1261-1267.

32. Azmi S, Jeziorska M, Ferdousi M, Petropoulos IN, Ponirakis G, Marshall A, Alam U, Asghar O, Atkinson $A$, Jones $W$ et al: Early nerve fibre regeneration in individuals with type 1 diabetes after simultaneous pancreas and kidney transplantation. Diabetologia 2019, 62(8):1478-1487.

\section{Tables}

Table 1. Demographic and clinical characteristics in subjects with mild cognitive impairment or dementia and no ischemia, subcortical ischemia and both cortical and subcortical ischemia. 


\begin{tabular}{|c|c|c|c|c|c|c|}
\hline & $\begin{array}{l}\text { No } \\
\text { ischemia } \\
(n=11)\end{array}$ & $\begin{array}{l}\text { Subcortical } \\
\text { ischemia } \\
(n=32)\end{array}$ & $\begin{array}{l}\text { Cortical \& } \\
\text { subcortical } \\
\text { ischemia } \\
(n=20)\end{array}$ & $\begin{array}{l}P \\
\text { value }^{1}\end{array}$ & $\begin{array}{l}P \\
\text { value }^{2}\end{array}$ & $\begin{array}{l}P \\
\text { value }^{3}\end{array}$ \\
\hline $\begin{array}{l}\text { Mild cognitive } \\
\text { impairment, } n(\%)\end{array}$ & $10(22.7)$ & $21(47.7)$ & $13(29.5)$ & $P$ value & $=0.25$ & \\
\hline Dementia & $1(5.3)$ & $11(57.9)$ & $7(36.8)$ & & & \\
\hline Age, years & $63.0 \otimes 7.3$ & $70.0 \rrbracket 8.8$ & $72.5 \bigotimes 7.5$ & $<0.05$ & $<0.01$ & 0.30 \\
\hline $\begin{array}{l}\text { Duration of } \\
\text { cognitive } \\
\text { impairment }\end{array}$ & $3.9 \otimes 5.9$ & $2.0 \bigotimes 1.9$ & $2.6 \rrbracket 2.3$ & 0.11 & 0.27 & 0.57 \\
\hline Female, n (\%) & $7(28.0)$ & $12(48.0)$ & $6(24.0)$ & $P$ value & $=0.17$ & \\
\hline Hypertension, n (\%) & $7(63.6)$ & $16(50.0)$ & $13(65.0)$ & $P$ value & $=0.51$ & \\
\hline Systolic BP, mmHg & $\begin{array}{l}137.9 凶 \\
18\end{array}$ & $135.8 \otimes 13.3$ & $141.1 \otimes 24.8$ & 0.69 & 0.66 & 0.28 \\
\hline Diastolic BP, mmHg & $\begin{array}{l}76.7 \rrbracket \\
8.6\end{array}$ & $73.3 \otimes 7.1$ & $73.9 \otimes 8.4$ & 0.24 & 0.28 & 0.99 \\
\hline Weight, kg & $\begin{array}{l}74.1 \otimes \\
19.3\end{array}$ & $82.4 \otimes 16.1$ & $72.6 \otimes 12.3$ & 0.17 & 0.79 & 0.05 \\
\hline $\mathrm{BMI}, \mathrm{kg} / \mathrm{m}^{2}$ & $\begin{array}{l}28.7 \rrbracket \\
7.4\end{array}$ & $31.8 \otimes 6.7$ & $27.3 \otimes 4.9$ & 0.17 & 0.56 & $<0.05$ \\
\hline $\mathrm{HbA} 1 \mathrm{c}, \%$ & $5.7 \otimes 0.5$ & $5.6 \otimes 0.6$ & $5.6 \otimes 0.3$ & 0.83 & 0.84 & 0.99 \\
\hline $\begin{array}{l}\text { Total cholesterol, } \\
\mathrm{mmol} / \mathrm{L}\end{array}$ & $4.8 \otimes 0.6$ & $4.9 \otimes 1.2$ & $5.2 \otimes 0.7$ & 0.89 & 0.38 & 0.34 \\
\hline $\begin{array}{l}\text { Triglycerides, } \\
\mathrm{mmol} / \mathrm{L}\end{array}$ & $1.1 \otimes 0.6$ & $1.4 \otimes 0.7$ & $1.5 \otimes 0.9$ & 0.34 & 0.20 & 0.60 \\
\hline \multicolumn{7}{|c|}{$\begin{array}{l}{ }^{7} \text { No ischemia versus subcortical } \\
\text { ischemia }\end{array}$} \\
\hline \multicolumn{7}{|c|}{$\begin{array}{l}{ }^{2} \text { No ischemia versus cortical \& subcortical } \\
\text { ischemia }\end{array}$} \\
\hline $\begin{array}{l}{ }^{3} \text { Subcortical ischemic } \\
\text { subcortical ischemia }\end{array}$ & ersus corti & & & & & \\
\hline
\end{tabular}

Data from 63 subjects presented as mean $\otimes$ standard deviation for numeric variables and frequency distribution for categorical variables. Continuous and categorical variables were compared using one-way ANOVA with LSD test for post hoc comparisons and Chi-square test, respectively. 
Table 2. Comparison of cognitive function, corneal nerve fiber morphology and volumetric brain MRI in subjects with mild cognitive impairment and dementia between no ischemia, subcortical ischemia and both cortical and subcortical ischemia.

\begin{tabular}{|c|c|c|c|c|c|c|}
\hline & $\begin{array}{l}\text { No } \\
\text { ischemia } \\
(n=11)\end{array}$ & $\begin{array}{l}\text { Subcortical } \\
\text { ischemia } \\
(n=32)\end{array}$ & $\begin{array}{l}\text { Cortical \& } \\
\text { subcortical } \\
\text { ischemia } \\
(n=20)\end{array}$ & $\begin{array}{l}P \\
\text { value }^{1}\end{array}$ & $\begin{array}{l}P \\
\text { value }^{2}\end{array}$ & $\begin{array}{l}P \\
\text { value }^{3}\end{array}$ \\
\hline MoCA & $24.3 \otimes 5.7$ & $20.8 \otimes 6.0$ & $18.8 \otimes 6.1$ & 0.10 & $<0.05$ & 0.28 \\
\hline CNFD, fibers $/ \mathrm{mm}^{2}$ & $31.2 \rrbracket 6.7$ & $27.0 \otimes 9.0$ & $21.7 \otimes 9.1$ & 0.18 & $<0.01$ & $<0.05$ \\
\hline $\begin{array}{l}\text { CNBD, } \\
\text { branches } / \mathrm{mm}^{2}\end{array}$ & $\begin{array}{l}78.3 \square \\
33.5\end{array}$ & $69.6 \otimes 45.4$ & $46.4 \otimes 28.5$ & 0.53 & $<0.05$ & $<0.05$ \\
\hline $\mathrm{CNFL}, \mathrm{mm} / \mathrm{mm}^{2}$ & $21.2 \otimes 5.1$ & $19.3 \otimes 6.9$ & $14.9 \otimes 5.8$ & 0.39 & $<0.01$ & $<0.05$ \\
\hline CNBD:CNFD ratio & $2.5 \otimes 0.9$ & $2.5 \otimes 1.2$ & $2.2 \otimes 1.4$ & 1.00 & 0.51 & 0.39 \\
\hline VPT, Volts & $\begin{array}{l}16.5 \otimes \\
13.4\end{array}$ & $18.3 \otimes 8.7$ & $18.6 \otimes 10.4$ & 0.65 & 0.61 & 0.93 \\
\hline ESC feet, $\mu S$ & $\begin{array}{l}62.4 \rrbracket \\
18.9\end{array}$ & $58.7 \otimes 18.5$ & $54.6 \otimes 20.4$ & 0.65 & 0.36 & 0.48 \\
\hline $\begin{array}{l}\text { Cortical gray } \\
\text { matter, ICV \% }\end{array}$ & $28.9 \otimes 3.5$ & $28.2 \varangle 3.8$ & $27.3 \llbracket 4.1$ & 0.63 & 0.29 & 0.42 \\
\hline $\begin{array}{l}\text { Hippocampus, } \\
\text { ICV \% }\end{array}$ & $0.47 \rrbracket 0.08$ & $0.43 \llbracket 0.08$ & $0.35 \llbracket 0.07$ & 0.24 & 0.001 & $<0.01$ \\
\hline Ventricle, ICV \% & $2.6 \rrbracket 2.0$ & $3.0 \bigotimes 1.9$ & $4.4 \bigotimes 1.3$ & 0.58 & $<0.05$ & 0.01 \\
\hline $\begin{array}{l}\text { Frontal lobe, ICV } \\
\%\end{array}$ & $10.6 \otimes 1.1$ & $10.2 \bigotimes 1.6$ & $9.6 \bigotimes 1.5$ & 0.50 & 0.11 & 0.20 \\
\hline $\begin{array}{l}\text { Temporal lobe, } \\
\text { ICV \% }\end{array}$ & $7.4 \llbracket 1.1$ & $7.4 \llbracket 1.1$ & $7.0 \otimes 1.2$ & 1.00 & 0.34 & 0.20 \\
\hline $\begin{array}{l}\text { Parietal lobe, ICV } \\
\%\end{array}$ & $6.8 \otimes 1.1$ & $6.2 \bowtie 0.9$ & $6.1 \bowtie 0.8$ & 0.10 & 0.07 & 0.76 \\
\hline $\begin{array}{l}\text { Whole brain, ICV } \\
\%\end{array}$ & $74.1 \rrbracket 3.1$ & $71.0 \rrbracket 4.6$ & $69.2 \bigotimes 3.2$ & 0.05 & $<0.01$ & 0.14 \\
\hline \multicolumn{7}{|c|}{$\begin{array}{l}{ }^{1} \text { No ischemia versus } \\
\text { subcortical ischemia }\end{array}$} \\
\hline \multicolumn{7}{|c|}{$\begin{array}{l}{ }^{2} \text { No ischemia versus cortical \& subcortical } \\
\text { ischemia }\end{array}$} \\
\hline $\begin{array}{l}{ }^{3} \text { Subcortical ischen } \\
\text { subcortical ischem }\end{array}$ & versus co & cal \& & & & & \\
\hline
\end{tabular}


Data from 63 subjects presented as mean $\otimes$ standard deviation for numeric variables and frequency distribution for categorical variables. Continuous and categorical variables were compared using one-way ANOVA with LSD test for post hoc comparisons and Chi-square test, respectively. Abbreviations: Montreal cognitive assessment (MoCA); Corneal nerve fiber density (CNFD); length (CNFL), branch density (CNBD), vibration perception threshold (VPT), electrochemical skin conductance (ESC) and intra cranial volume (ICV).

\section{Figures}
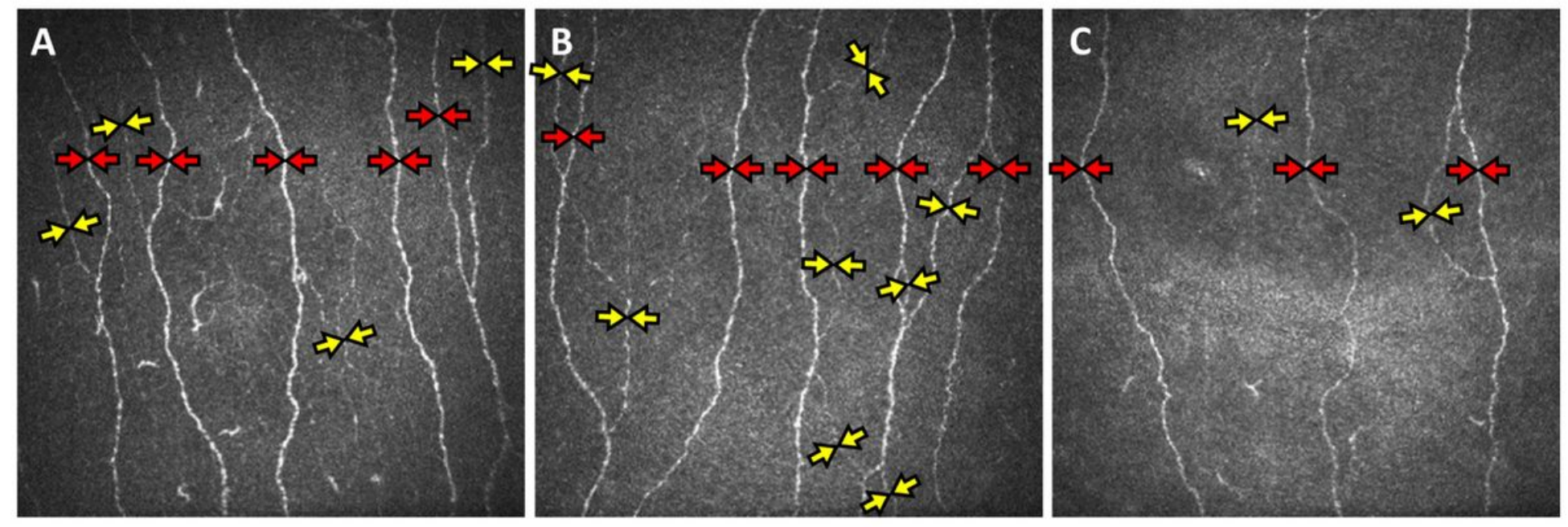

\section{Figure 1}

Corneal confocal microscopy (CCM) images of the sub-basal nerve plexus of subjects with mild cognitive impairment without cerebral ischemia (A), with subcortical ischemia (B) and cortical and subcortical ischemia (C) showing reduced main nerve fibers (red arrows), branches (yellow arrows) and total fiber length in subjects with cortical and subcortical ischemia. 

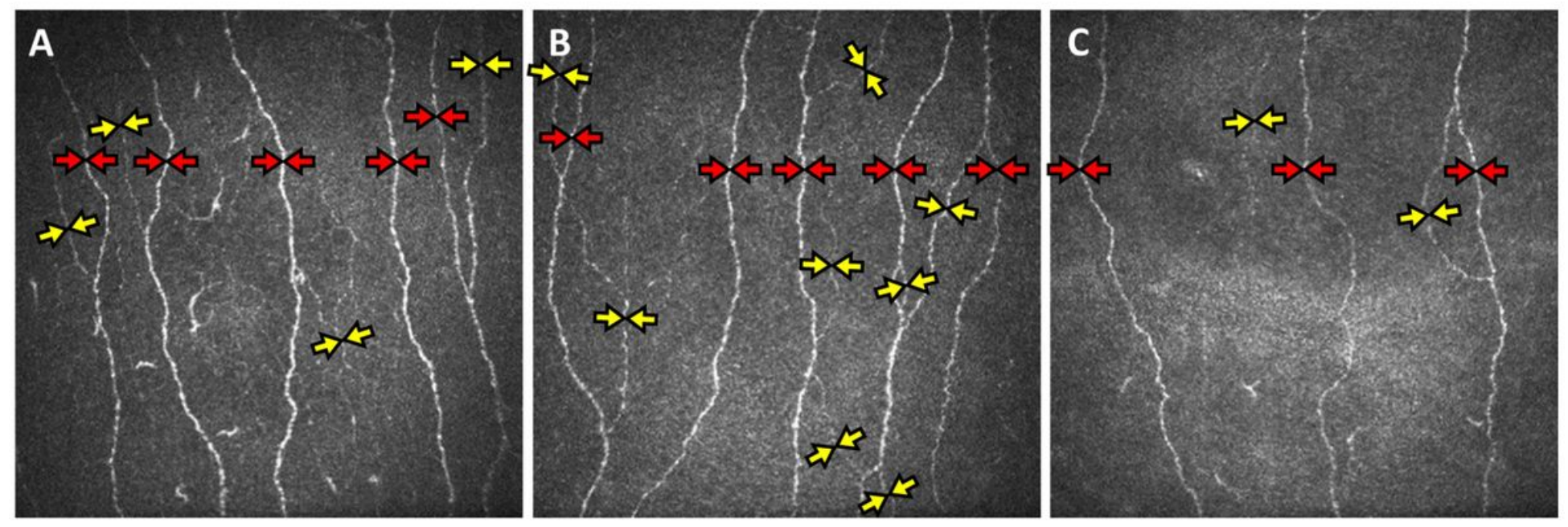

\section{Figure 1}

Corneal confocal microscopy (CCM) images of the sub-basal nerve plexus of subjects with mild cognitive impairment without cerebral ischemia (A), with subcortical ischemia (B) and cortical and subcortical ischemia (C) showing reduced main nerve fibers (red arrows), branches (yellow arrows) and total fiber length in subjects with cortical and subcortical ischemia. 


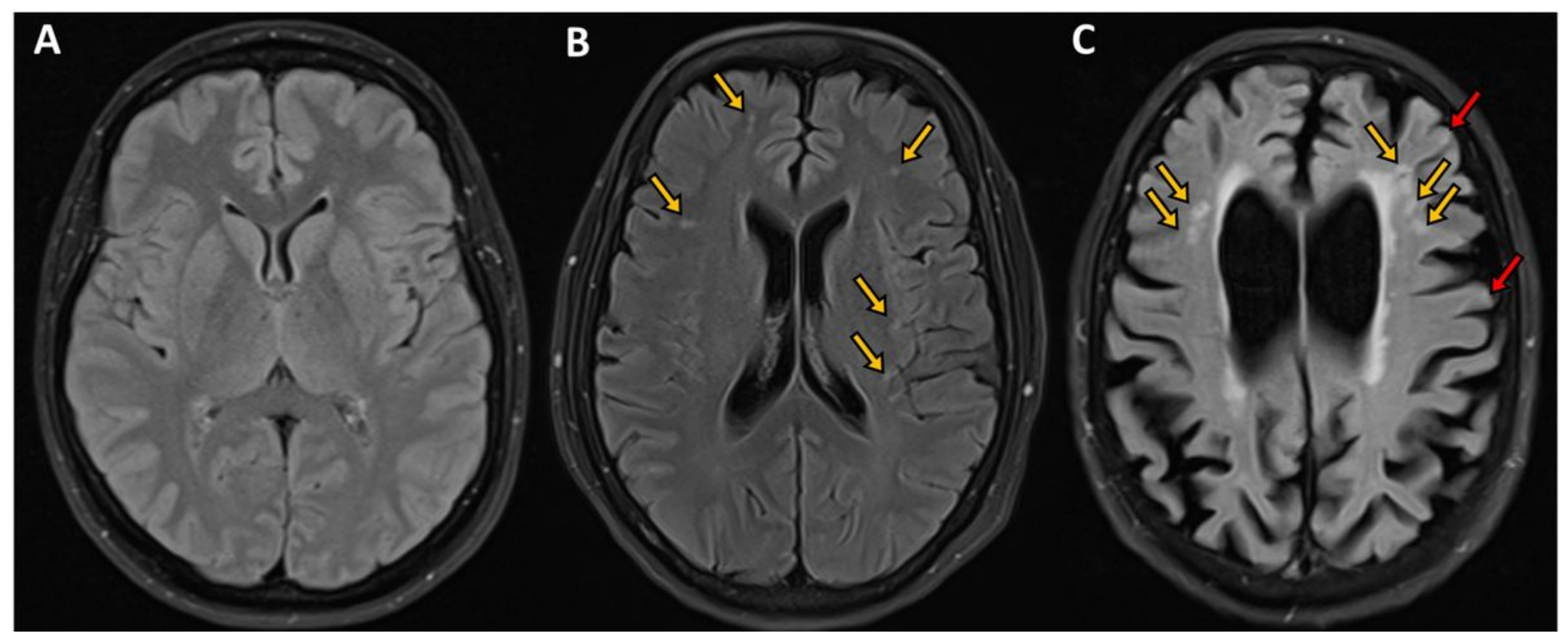

Figure 2

Axial FLAIR images of the brain of subjects with mild cognitive impairment showing A. normal appearing visualized brain parenchyma with no evidence of ischemia, infarction or microbleeds, B. bilateral scattered subcortical white matter ischemic changes in the form of hyperintense (bright foci) secondary to microangiopathy shown in orange arrows and C. moderate brain atrophy with cortical and subcortical ischemic foci in the form of bright signal intensity shown by orange and red arrows, respectively. Courtesy of Dr. Ahmed Elsotouhy.

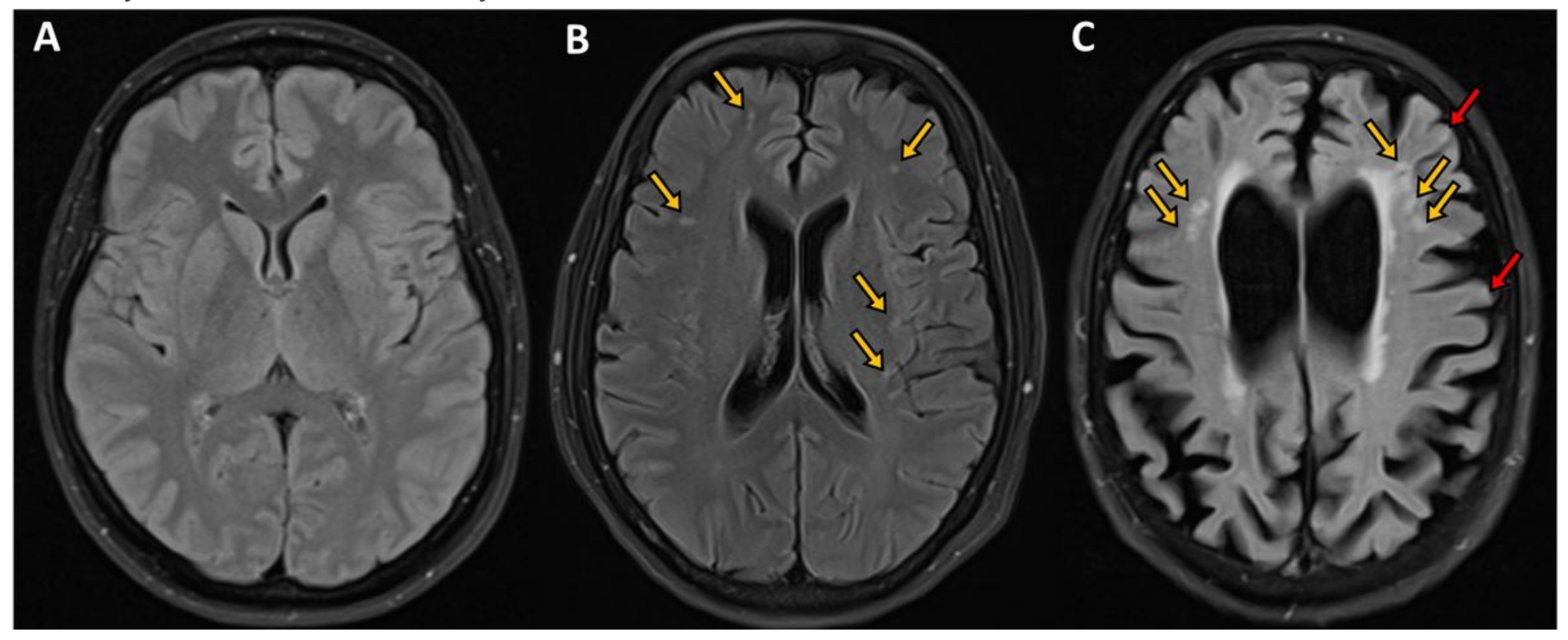

Figure 2

Axial FLAIR images of the brain of subjects with mild cognitive impairment showing A. normal appearing visualized brain parenchyma with no evidence of ischemia, infarction or microbleeds, B. bilateral scattered subcortical white matter ischemic changes in the form of hyperintense (bright foci) secondary to microangiopathy shown in orange arrows and C. moderate brain atrophy with cortical and subcortical 
ischemic foci in the form of bright signal intensity shown by orange and red arrows, respectively. Courtesy of Dr. Ahmed Elsotouhy. 